\title{
12 Beantworten von Gutachterkommentaren
}

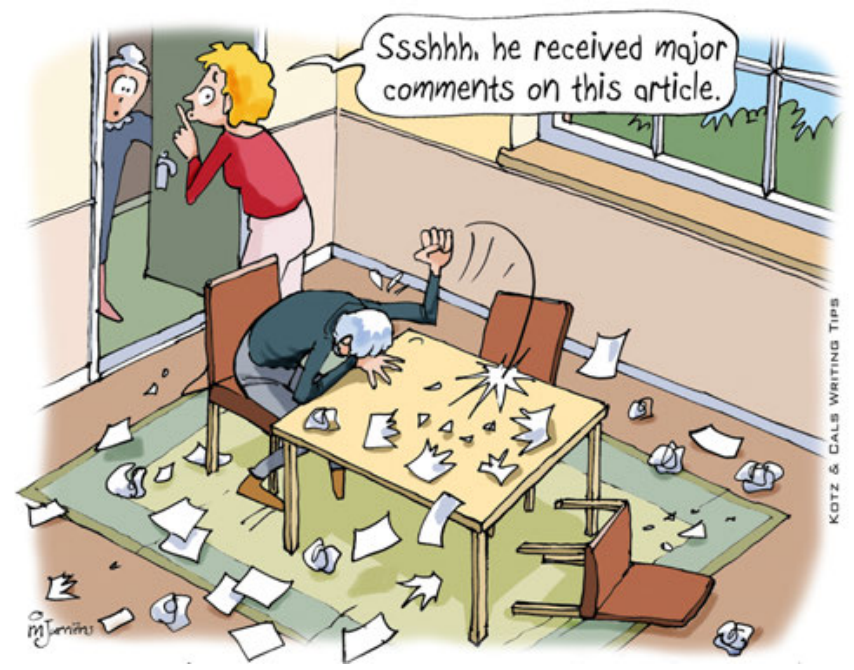

Tipp 12 - Beantworten von Gutachterkommentaren: immer locker bleiben!

\section{Was Sie wissen sollten}

Es gibt im Wesentlichen drei redaktionelle Entscheidungen über eingereichte Manuskripte: Annahme, Ablehnung (direkte Ablehnung durch den Zeitschriftredakteur oder Ablehnung nach Gutachterkommentaren) und Überarbeitung anhand von Gutachterkommentaren. Viele veröffentlichte Artikel wurden mehrmals abgelehnt und überarbeitet, bevor sie letztendlich angenommen wurden. Die Entscheidung zur Überarbeitung und neuerlichen Einreichung ist ein Zeichen dafür, dass eine Zeitschrift grundsätzlich an einem Manuskript interessiert ist. Somit besteht eine gute Chance zur Annahme, wenn die Kommentare der Gutachter zufriedenstellend beantwortet werden.

Sogenannte „peer reviewed“ Zeitschriften haben die Aufgabe, von mindestens zwei unabhängigen Experten (,peers“) aus dem gleichen Fachgebiet wie das der Autoren mindestens zwei hochwertige Gutachten („reviews“) zu erhalten. Solche Gutachten enthalten Kommentare und Empfehlungen für die Autoren (normalerweise anonym) und zusätzliche Anmerkungen für den Redakteur, die nicht an die Autoren weitergeleitet werden. Dabei ist $\mathrm{zu}$ beachten, dass sich die Kommentare und Empfehlungen der Gutachter häufig voneinan- 
der unterscheiden. Der Redakteur muss anhand der Gutachten beurteilen, ob die in einem Manuskript dargestellten Forschungsergebnisse hinreichend gesichert sind, und ob diese neu und relevant für die Leserschaft sind.

Die Entscheidungen, ein Manuskript nach Begutachtung abzulehnen (,,reject with review“) oder nach Überarbeitung erneut einzureichen (,revise and resubmit“) enthalten die Kommentare der Gutachter und manchmal zusätzliche Kommentare des Redakteurs. Ein hochwertiges Gutachten ist unterteilt in Hauptkommentare („major comments“), die bei einer Überarbeitung unbedingt und sehr sorgfältig beantwortet werden müssen, und Nebenkommentare (,minor comments"), die auch beantwortet werden müssen, aber weniger entscheidend für die abschließende Beurteilung sind. Jeder einzelne Kommentar umfasst idealerweise einen klaren Kritikpunkt hinsichtlich eines spezifischen Teils des Manuskripts und eine Anregung zur Überarbeitung (falls möglich). Die überarbeitete Version des Manuskripts wird vom Redakteur gelesen und oftmals an die gleichen Gutachter zurückgegeben, um festzustellen, ob alle Kommentare und Empfehlungen zufriedenstellend adressiert wurden. Gutachter und Redakteur können dann um weitere Überarbeitungen bitten.

\section{Was Sie tun sollten}

Verfallen Sie nicht in Panik, wenn Ihr Manuskript nach Begutachtung abgelehnt wird! Trösten Sie sich mit dem Wissen, dass Manuskripte häufiger abgelehnt als angenommen werden. In den Gutachterkommentaren sollten Sie kostenlose Ratschläge zur Verbesserung Ihres Manuskripts finden. Lesen Sie diese, schlafen Sie drüber und lesen Sie sie dann erneut, um über die Gründe für die Ablehnung nachzudenken und daraus zu lernen. Teilen Sie die Entscheidung und die Gutachterkommentare so bald wie möglich Ihren Koautoren mit und nutzen Sie die Gelegenheit, Ihr Manuskript damit weiter zu verbessern, bevor Sie es an eine andere Zeitschrift senden. Lassen Sie nicht zu viel Zeit verstreichen und motivieren Sie sich dazu, so schnell wie möglich mit dem erneuten Einreichungsprozess zu beginnen. Gehen Sie dabei genauso gründlich vor wie beim ersten Mal (siehe Teil 11: Einreichen eines Artikels).

Wenn Sie die Entscheidung erhalten, Ihr Manuskript zu überarbeiten und erneut einzureichen, dann sollten Sie erst die Gutachterkommentare sorgfältig lesen und auf sich wirken lassen, bevor Sie eine Antwort verfassen. Kopieren Sie zunächst alle Kommentare wortwörtlich in ein neues Dokument und beantworten Sie jeden einzelnen Kommentar gemäß der folgenden zwei Schritte: (1) Antwort der Autoren (,,authors' response“): Beantworten Sie kurz den Kommentar (diese Korrespondenz zwischen Gutachtern und Autoren erscheint später 
nicht im Artikel); (2) Änderungen am Artikel („,changes to the manuscript“): Geben Sie an, ob und wo im Manuskript Sie etwas geändert haben. Geben Sie Überarbeitungen Ihres Artikels im Präsens oder im Perfekt an, z. B. „Wir präsentieren jetzt Daten zu [...] in Tabelle 1“ oder „Wir haben im dritten Absatz des Abschnitts Methoden Informationen zu [...] hinzugefügt“. Markieren Sie in Ihrem überarbeiteten Manuskript den Text, der gegenüber der vorherigen Version geändert wurde (hierzu eignet sich sehr gut der Änderungsmodus („,track changes“) Ihres Textverarbeitungsprogramms). Wenn Sie Entwürfe Ihrer Antworten auf die Gutachterkommentare und Ihres überarbeiteten Manuskripts fertiggestellt haben, schicken Sie beide Dokumente an die Koautoren. Integrieren Sie deren Änderungsvorschläge in einer finalen Version, und holen Sie sich das Einverständnis der Koautoren, diese bei der Zeitschrift einzureichen.

Seien Sie gegenüber den Gutachtern bei Ihrer Antwort auf deren Kommentare immer respektvoll. Bedanken Sie sich für Verbesserungsvorschläge, und versuchen Sie, so viele Anregungen wie möglich zu berücksichtigen. Sie können einem Gutachterkommentar jedoch auch respektvoll widersprechen. Untermauern Sie dabei Ihren Gesichtspunkt mit soliden Argumenten, indem Sie Verweise $\mathrm{zu}$ Belegen aus Ihren eigenen Daten oder zu veröffentlichten Arbeiten einbeziehen. Einige Kommentare können in der Antwort des Autors adressiert werden, ohne Änderungen am Artikel vorzunehmen, vor allem, wenn es keine spezifische Anregung zur Überarbeitung vonseiten des Gutachters gab. Auf alle Fälle sollten Gutachter, die Ihre Antwort und den überarbeiteten Artikel lesen, den Eindruck erhalten, dass Sie deren Kommentare ernst genommen haben und Ihr Bestes getan haben, um den Artikel entsprechend zu verbessern. Wenn alles gut verläuft werden Sie schließlich die erhoffte E-Mail erhalten, in der man Sie zur Annahme des Artikels beglückwünscht. Genießen Sie diesen Moment und feiern Sie die Veröffentlichung! 


\section{Checkliste für Beantworten von Gutachterkommentaren}

- Geben Sie Punkt für Punkt eine Antwort auf alle Gutachterkommentare, strukturiert als:

- Antwort der Autoren an den Gutachter (in respektvollem Ton)

- Änderungen am Artikel (ob/welche und wo)

- Stellen Sie eine markierte Überarbeitung Ihres Artikels zur Verfügung

- Im Fall einer Ablehnung:

- $\quad$ seien Sie nicht enttäuscht und motivieren Sie sich selbst, zügig fortzufahren,

- verbessern Sie Ihren Artikel basierend auf den Kommentaren der Gutachter, so weit wie dies möglich ist, und

- reichen Sie die neue Version rasch bei einer anderen Zeitschrift ein.

- Holen Sie sich das Einverständnis Ihrer Koautoren zu Überarbeitungen und neuen Einreichungen 
